\title{
Exploration of Potential Energy Savings from Smart Appliances
}

Natascha S. Castro 


\title{
Exploration of Potential Energy Savings from Smart Appliances
}

\author{
Natascha S. Castro \\ U.S. DEPARTMENT OF COMMERCE \\ National Institute of Standards and Technology \\ Building Environment Division \\ Building and Fire Research Laboratory \\ Gaithersburg, MD 20899-8631
}

Prepared for: U.S. Department of Energy

July 2005

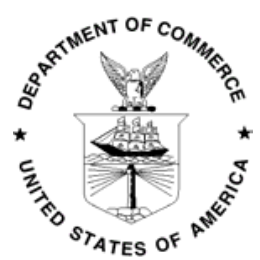

U.S. DEPARTMENT OF COMMERCE Carlos M. Gutierrez, Secretary TECHNOLOGY ADMINISTRATION Michelle O'Neill, Acting Under Secretary of Commerce for Technology NATIONAL INSTITUTE OF STANDARDS AND TECHNOLOGY 


\begin{abstract}
Technological innovations coupled with the decreasing costs of sensors have brought about a new generation of "Smart" appliances. While current sensor-based appliances have shown the potential to offer consumers increased options for energy and cost savings by optimizing energy use for improved performance, smart appliances have the potential to process internal operation information and the factors that can influence appliance efficiency (i.e., load and weather data, and utility pricing information.) Both in the United States and internationally, advances in sensor technology are rapidly changing product capabilities and many innovations have moved from concept or prototype stage to market.
\end{abstract}

In FY 2005, NIST completed a study of existing home networking technologies for smart appliance applications. The purpose of this study was to determine the communications infrastructure needed to bring more efficient appliance applications to market and to evaluate the benefits of selected technologies, highlighting opportunities for advancing more energy efficient appliances.

This report provides an overview of recent innovations in residential appliances and identifies specific appliances with enhanced energy savings potential in home-networked environments.

\title{
Acknowledgments
}

This work was supported by the U.S. Department of Energy, Office of Energy Efficient and Renewable Energy.

Certain trade names or and industry standards are mentioned in the text to illustrate statements. In no case does such identification imply recommendation or endorsement by the National Institute of Standards and Technology, nor does it imply that the technology is the best available for the purpose. 


\section{Introduction}

According to data from the U.S. Department of Energy (DOE), residential buildings use over $20 \%$ of the energy consumed in the United States, generating an estimated 313.4 metric tons of carbon monoxide annually. Furthermore, national survey data for 2001 showed that $31 \%$ of the residential energy consumption was attributed to home appliances and air-conditioners [1]. Historically, government and industry have recognized these as significant energy loads and worked to identify ways to reduce energy consumption for residential appliances. The need for this work is amplified in light of increasing energy demand and limited resources.

In 1975, the Energy Policy and Conservation Act (EPCA) established an energy conservation program for major household appliances. DOE's Office of Energy Efficiency and Renewable Energy, Buildings Technology Program conducts the program, which addresses testing, labeling, and education [2]. EPCA was expanded in 1978 to address federal energy conservation standards and to include certain industrial equipment. Overall it has had a tremendous impact, promoting energy efficiency measures for covered products [3].

The Department of Energy's list of residential "covered products" encompasses all the residential appliances for which there is a federal test procedure to rate energy consumption as well as a federal minimum energy standard. This includes: refrigerators/freezers, room and central air conditioners, heat pumps, water heaters, furnaces, dishwashers, clothes washers, clothes dryers, kitchen ranges and ovens, pool heaters, television sets, fluorescent lamps and ballasts, incandescent reflector lamps, faucets, showerheads, water closets and urinals. This report focuses on the primary energy-using appliances typically found in U.S. households with the aim of identifying recent innovations and technological improvements, including those still at the prototype stage.

The report begins with an overview of existing home communications technologies and then presents the status of developments for industry protocol and smart home platforms internationally and in the United States. The next section addresses innovative applications and industry trends with specific information for refrigerators, laundry machines, dishwashers, water heaters, ranges and ovens and presents scenarios for energy savings using home networking. The report then provides conclusions and recommendations.

\section{Overview of Communication Technologies}

Traditionally the consumer's home electrical system has been used to power individual appliances as well as to provide electricity to lighting fixtures and outlets throughout the home. In recent years, this wiring system has been extended to provide additional communications capabilities, and alternatives including the use of phone lines, radio signals (wireless), infrared, and communications wiring have been tested. The tradeoffs for these technologies are summarized below. 
Powerline carrier (PLC) networks transmit data (i.e., voice, faxes, e-mail, and Web pages) over regular electrical wiring. The advantage is that virtually every home is already equipped with a power line network, transforming every electrical outlet in the immediate area into a network connection. When properly applied, wires and sockets are used simultaneously for electricity and data, without disrupting each other. Hence, appliances designed with unique identifications such as individual Internet protocol (IP) addresses can be connected to the power line network, giving access to each appliance. The current industry standard for PLC is HomePlug 1.0, developed by the HomePlug Powerline Alliance, an industry consortium [4]. This technology seeks to bridge home power lines with existing network technology.

Phone lines, also pre-existing in most homes, have the added benefit that the wire is already suitable for voice communications and data transfer. The use of this technology has already been extended to provide secure, digital distribution of video, music and data to all connected rooms in a home. However, the disadvantages are that additional wiring may be necessary to increase the number of phone connections and interference is a real problem because phone wires are typically not shielded. Static noise on the phone line, as well as changes in weather and electrical storms, can also cause phone line problems.

Infrared is a reliable and inexpensive communications option widely used for remote control of televisions; however, its primary disadvantage is that it only works on line of sight, which therefore limits its use in a smart home. It also has the disadvantage of limiting communication to between only two devices at one time, which may not be practical when trying to connect several appliances on a network.

The use of radio frequency $(\mathrm{RF})$ signals for wireless networking applications has been marketed in the past decade with great acceptance in the computer industry and is now more widely applicable. The Institute of Electrical and Electronics Engineers, Inc.(IEEE) established early standards for wireless communications including: IEEE 802.11 (Wi-Fi) standards that support air interface between and among radio-frequency wireless clients in local area networks and IEEE 802.15 standards, which provide for low-complexity, low power wireless connectivity for portable devices in wireless personal area networks [5]. The technology is under continued development to optimize designs for specific application areas (i.e., home control and monitoring, building automation, industrial applications). In the United States and internationally, consortiums of manufacturers have evaluated existing wireless communications options and have worked together to develop industry protocol to establish a basis for new networked product lines that offer interoperability based on the IEEE standards. Two examples are described below:

1) a proprietary RF wireless communications technology designed for residential and light commercial control and status reading applications.

2) an interoperable standards-based RF wireless communications technology designed for industrial, residential, and home control and monitor applications.

Other structured communications wiring options can provide the means to create a home network. This includes RG-6 (coaxial) wire typically used for entertainment 
systems, CAT 5 (network cable) wire for data and communications, or special cables for home control or fiber optics [6].

\section{Industry Protocol and Smart Home Platforms}

In the appliance industry there is no 'standard home network'; rather, the trade-offs for various technologies must be weighed to find the appropriate solution for different applications. Several associations have worked together to create and industry protocol that addresses the needs associated with providing added functionality to common appliances to creating new ways to improve the home environment.

In 1988, the Electronic Industries Association of Japan published two home networking protocols, ET 2101 and JEM 1439. These standards are now available through the Japanese Electronics and Information Technology Industries [7]. More recently, a group of companies established a technical specification to facilitate the development of home networking systems by creating a platform that requires no special rewiring, so that it can be readily applied to existing homes and can easily control a wide range of devices. The result is the Energy Conservation and Homecare Network (ECHONET) Standard version 1.0 published in 2000 and version 2.0 published in 2001 [8]. This protocol is the basis for the development of smart home platforms. Lessons learned in Japan are applicable in the United States. By developing the standard industry protocol, individual companies were able to focus their resources on developing network compatible devices, rather than having to develop new platforms. The added product capabilities already increase the complexity of the appliances and present a challenge and therefore a cost to developers.

One manufacturer introduced a networked home appliance system in late 2003. This platform is used to control networked home appliances using wireless control (via terminal or phone) and can also receive information services via the Internet. These services include centralized control and monitoring of appliances such as operational status, maintenance issues, and energy conservation guidelines; watchdog alarming services to alert for intruders or unlocked doors; and transmission of useful information such as weather, news, etc.

In December 2003, the European Committee of Manufacturers of Domestic Equipment (CECED), an association of over 200 industrial companies in the appliance sector, launched an interoperability platform for European household appliances. The new platform, called CHAIN (CECED Home Appliance Interoperating Network), provides a means of integrating all household appliances to a virtual network by using their existing communications protocol (EHS/Konnex and LonWorks) and proprietary control systems [9]. The platform enables the control and automation of key services in a home such as remote control of appliance operation, energy or load management, remote diagnostics and automated maintenance support to appliances, and downloading and updating of data, programs, and services from the web.

In the same year, the Association of Home Appliance Manufacturers (AHAM) published an American National Standards Institute/AHAM standard on Connected Home Appliances-Object Modeling [10]. This standard described generic appliance models, 
objects, and high-level messages as a means to enable new appliance services and features through networking. The standard is written for appliances that contain a communications interface module linked to a home network system. Standardized elements of appliances are then accessible and can be controlled remotely by users, service providers, and other devices, independent of the underlying network.

The overall trend is to form consortiums, teaming appliance manufacturers with technology companies to develop and promote products. In the case of enabling technologies, consortia include: Home Phone Network Alliance, Homeplug Power Alliance, Wi-Fi Alliance and the Internet Home Alliance. These are generally developed as non-profit associations of high-tech and consumer companies with the goal of breaking down barriers for market acceptance through education and coordinated product development. In addition, some companies are developing ways to combine existing technologies for new networking capabilities. One home control network protocol introduced in 2004 is based on a combination of RF and Powerline communication technologies.

\section{Innovative Applications and Industry Trends}

Industry and consumer organizations have increased consumer awareness of multifunction, 'smart' appliances enhanced with innovative sensors and communication technologies and have highlighted their potential to increase functionality and optimize energy use. Similar efforts should be made to educate consumers on the benefits of environmentally friendly designs that facilitate maintenance, disassembly and recycling.

The appliance industry has a wealth of innovative ideas, yet market barriers have kept many products at the prototype or concept stage. Several major companies invested in the development of innovative appliances that could offer added functionality using home networking and/or Internet access; however, both internationally and in the United States, the market penetration of networked appliances has been relatively small. In fact, the most successful products are not marketed as web-appliances for fear that consumers might be alienated. In contrast, the market penetration of appliances with improved sensor technology has increased steadily. The following sections will introduce recent product developments for some energy-intensive "covered" products.

\section{Refrigerators, Freezers, and Wine Chillers}

Refrigerators are under constant development as manufacturers seek to find new features that could appeal to consumers, including home networking, advanced control logic, temperature control and component improvements.

In 1999, a major manufacturer developed a prototype Internet refrigerator that was used in field tests; however, six years later, the appliance remains a prototype. In 2001, a similar product, a prototype web-enabled refrigerator, was introduced. Because the consumer demand was not high, versions of this prototype remain in the development and demonstration phase with an on-going field test in 20 homes. In 2003, another manufacturer debuted its version of the Internet enabled refrigerator. In the following year, yet another manufacturer introduced its digital multimedia refrigerator, a product 
that provides a fixed Internet connection for entertainment, access to cooking information, memory storage, and maintenance alerting capabilities. This technology primarily provides added convenience and functionality but it does not exhibit a significant energy savings potential. Indirectly there are potential energy savings when the technology alerts the consumer or a service company of operational faults that could otherwise result in increased energy use if undetected.

The two most significant energy-saving innovations for refrigerators are: 1) the development of a more efficient defrost algorithm by Manufacturer A in 2002 and 2) the development of improved compressor technology that was first introduced in a residential refrigerator in 2004 by Manufacturer B. Manufacturer A's defrost algorithm, a long-time automatic defrost, improved on previous approaches by modifying the start/stop of the defrost cycle relative to the compressor cycle and resulted in the need for a change to the DOE test procedure [10]. The original practice was to go straight from compressor cooling to defrost heating. However, allowing the coils to reach the refrigerator temperature before starting the defrost heater enables the efficient use of the thermal mass of the refrigerator to 'pre-heat' the coils at no cost. Similarly, waiting to start the compressor after the defrost heater turns off allows the coils to cool down to the refrigerator temperature. Reducing the duration of defrost cycles resulted in up to $75 \%$ energy savings. Manufacturers now commonly use this approach, a clear energy saver. The second development, by Manufacturer B, uses linear compressors to provide more energy-efficient cooling. Instead of the typical reciprocating compressor found in most refrigerators, the new compressor design eliminates the need to change the rotary motion into a reciprocating motion, which reduces the compressor's energy loss and improves the motor-efficiency system. When tested in a refrigerator, Manufacturer B reported a $47 \%$ reduction in energy consumption.

Other recent advances are aimed at improving the temperature control for specific levels or compartments. Improved control algorithms can also improve energy efficiency. One manufacturer introduced a control system, which uses variable speed fans and temperature sensor information to deliver the appropriate amount of cool air to different locations. This approach has also been introduced in high-end wine storage systems to provide multi-zone cooling capabilities. These high-end, niche products are just beginning to have broader appeal.

\section{Laundry Machines}

Advances in sensor technology have had a significant impact on wash efficiency, improving cleaning performance while reducing energy and water consumption. New products take advantage of fabric sensing, load sensing, and fill control capabilities in washers and moisture sensing in dryers. In addition, some clothes washers offer an onboard heater for improved cleaning performance and run at higher spin speeds for more efficient water extraction.

Manufacturers are providing consumers with improved cycle options for better cleaning performance and fabric care. This includes a larger array of wash cycles customized for specific fabrics (i.e., silk, cashmere, wool) as well as guides options for treating stains. 
One manufacturer developed a pre-treatment cycle that mixes water and detergent to create a concentrated cleaning lather to saturate the clothes in a spinning cycle before the normal wash cycle begins. Another product trend is the increased offering of horizontal axis machines. Horizontal axis machines inherently have dramatically lower water consumption and are often operated with much higher spin speeds, which result in a tremendous energy savings for both washing and drying. One drawback for consumers is that regardless of the load size, the drum size in horizontal models is smaller and therefore loading is more difficult than conventional, vertical axis machines. However, consumers are growing increasingly accepting of horizontal axis models after weighing the advantages.

Manufacturers have also worked to introduce networked clothes washers. In Europe, in 1999, a high-tech clothes washer that integrated a wireless modem was introduced. At a retail price of $\$ 1200$, more than double the price of conventional models, this innovative product was not well received. The manufacturer improved its approach the following year and introduced a version that uses a power-line modem to provide appliance information for networking at a price comparable to conventional models. In Japan, in 2004, a manufacturer introduced a washing machine that communicates via Bluetooth with a computer within a $9.1 \mathrm{~m}(30 \mathrm{ft})$ radius to download clothing care instructions and will also tell a PC when it needs maintenance. Several U.S. companies have also made significant investments in developing internet-enabled/home networked washing machines, but these products have not yet entered the market.

A different form of appliance networking has been introduced in the United States. One manufacturer provided a linked washer and dryer that communicate to synchronize drying times with washer run times, while another manufacturer developed a product that programs the dryer setting based on sensor measurements from the clothes washer.

Other innovations address clothes dryers. In 2003, a novel concept for a combined appliance was introduced. The appliance is a drying cabinet mounted over a conventional tumble dryer. It has a secondary heating element and blower but operates using the same vent and power supply as the tumble dryer. This product is designed to improve drying of articles that cannot handle the tumble dry environment (i.e., sweaters, shoes). In the same year another manufacturer introduced a stand-alone unit designed for specialty drying. For conventional dryer models, a design change meant to reduce wear on clothing is increased drum size. This also has the potential to improve drying times due to better air circulation.

\section{Dishwashers}

Adaptive control dishwashers with soil-sensing capabilities have been on the market in the United States for nearly a decade. However, their market share has increased dramatically in recent years along with advances in control algorithms that incorporate new sensor capabilities. These machines have the potential for energy savings because the control algorithms are designed to shorten the wash cycle when the sensors indicate a low soil load. Although new models offer temperature control that would result in increased energy use, advances in the optimization of water use based on soil levels and 
load size could produce a net energy savings. In addition, water hardness sensors have been introduced to optimize the use of detergent.

The other trend in manufacturer's products is the development of large capacity dishwashers. Design advances including the development of a horizontal motor, improved wash-arm technology, and more efficient dish rack layouts have enabled manufacturers to increase the loading capacity of some dishwasher models. Although the exterior dimensions of dishwashers are unchanged, the internal tub size for many highend models has increased and loading flexibility has improved. Some models offer adjustable rack heights, three loading levels, and loading supports that can be moved out to accommodate larger items [12]. The result is that the super-capacity dishwashers can hold as many as 14 to 16 place settings, whereas conventional dishwashers hold 10 to 12 place settings. These super-capacity dishwashers are readily available in the U.S. market.

\section{Water Heaters}

Although the use of smart controls in water heaters is relatively new and limited, the energy savings are significant and could be a major driving force for this technology. These innovations include the introduction of the industry's first programmable electric water heater in 2000 , which was estimated to cut electrical operating costs by $15 \%$. In 2001, a smart water heater, with adaptive electronic controls, was introduced. It has a self-diagnosing control system that monitors the water heater's critical functions and modulates the setpoint temperature based on consumption. The purpose of the setpoint optimization is to reduce standby losses; this product reportedly cuts standby losses by $30 \%$ over a standard water heater. Other advances for water heaters include improved insulation and improved corrosion resistance to extend product life.

\section{Ranges and Ovens}

Developments in ranges and ovens have focused on increasing the thermal output, providing additional burners to approximate commercial stoves, and adding convenience items such as warming drawers. In contrast, the most innovative product in this area is a refrigerated range, which augments a conventional range with refrigeration capabilities. This combination product is marketed for its convenience since it can be preprogrammed for a cooling period followed by a cooking period. All of these features represent convenience at a net increase in energy consumption.

\section{Potential for Energy Savings Scenarios from Networked Home Appliances}

Energy savings are currently secondary to the convenience features that often drive consumer purchases; however, as consumers become more aware of increasing energy costs, the long-term savings will play a larger role.

In the case of home networking, the network of appliances can be linked to an Internet port. The use of system sensors combined with direct access to the information enables remote monitoring of the system status, remote control of appliances, and thus provides increased security (i.e., knowing for certain that an appliance was not left on). Some scenarios for energy savings include remote setpoint adjustment for water heater 
temperatures (i.e., for vacation mode) and Internet access to relevant product use information.

The key to energy savings is time of use. As utilities offer greater incentives to shed peak loads (i.e., reduced rates for off-peak use), households could take advantage by remotely operating their appliances and shifting loads to off-peak hours. Certain appliances such as dishwashers and water heaters can be programmed to run in off-peak hours and the energy savings is evident to the consumer. In addition, weather forecasting data could be used as a valuable input to the decision process in an internet-enabled thermostat. By taking into account near-term weather changes in conjunction with peak utility rates, the thermostat setpoint could be optimized rather than running on a preset time of day schedule.

Other potential savings arise from the capability of receiving information about necessary maintenance actions or required repairs if a fault has been detected. With the increasing use of sensors, a networked appliance can share operational information with a central 'brain'. Fault detection algorithms can be used to determine if maintenance is needed. This can be particularly useful for appliances that are energy-intensive. Appliances can experience degradation in energy efficiency long before a consumer detects a drop in performance. For example, if a refrigerator coil is fouled, the cooling capacity of the coil is reduced and hence, in order to maintain the internal setpoint temperature, the compressor run times would be increased. At the extreme, the refrigerator would not be able to meet the internal setpoint temperature. Similarly, if system temperatures are indicative of a refrigerant leak, the owner or a service company could be contacted.

\section{Summary and Recommendations}

This report presents the results of an FY2005 scoping study to explore the potential energy savings from smart appliances.

Home networking is an enabling technology that has been under development for several years internationally, and more recently in the United States. Industry has formed various consortia to promote the development of standard protocols for specific communications technologies, including the Homeplug Power Alliance, Home Phone Network Alliance Wi-Fi Alliance for powerline, phoneline, and wireless communications technologies, respectively. This collaborative work complements individual product development. Internationally, home networking standards have already been developed and industry is working to develop their product lines to incorporate new functionality. In the United States there is also a standard on connected home appliances, but industry is just beginning to introduce smart platforms and home networked products; consumer demand is still low.

The main technological innovations for residential appliances are aimed at: the efficient use of sensors, Internet enabling, and the development of more environmentally friendly designs. For individual appliances, the thrust is different based on consumer demands. The appliance industry is also taking a proactive role in helping to educate consumers on 
the benefits of the various technologies. Many of the products described in this paper are in prototype stage or have recently been introduced to the consumer market.

For refrigerators, Internet enabling has generated little consumer acceptance. Improved temperature control for compartments may improve the efficient use of cooling, but the most significant energy-saving improvements come from the creation of a more efficient defrost algorithm and the introduction of linear compressor technology for a $47 \%$ reduction in energy use.

For laundry machines and dishwashers, the energy-saving innovations are linked to improved use of sensors for fabric sensing, load sensing, fill control, and moisture sensing in dryers. This allows for cycle optimization and reduces the occurrence of overwashing or over-drying. For clothes washers, appliance networking appears to be successful on a small scale (e.g., linking clothes washer to clothes dryer so that embedded logic can select the proper cycle). Another trend, though not a new technology, is the increased production of horizontal axis machines for the U.S. market. Consumers are weighing tradeoffs between loading convenience and energy efficiency and more and more are choosing horizontal axis machines for their energy efficiency.

For water heaters, the use of smart controls is promising. It is estimated that the electrical energy operating costs can be reduced by $15 \%$ through the use of programmable thermostats and that standby losses can be reduced by $30 \%$ through the use of setpoint optimization.

In contrast, innovative products for ovens and ranges offer convenience improvements at a net energy increase. These include 5-burner and 6-burner models with increased thermal output, warming compartments, and in one case, refrigeration capabilities in addition to programmable oven start and stop times.

Home Networking has the potential to reduce energy consumption in addition to providing added functionality and convenience. The key advantages are summarized below:

* Time of Use- the ability to control when the appliance cycle is run, either preset or operated remotely for convenience and access to off-peak utility rates where available

* Maintenance Alerts-the capability of receiving information about necessary maintenance actions or required repairs if a fault has been detected

* Internet Access- to relevant product use information

One area of research that is not well publicized is the development of environmentally friendly technologies (i.e., design of easily recyclable components). Because manufacturers are constantly looking to improve the functionality of their products and to address changing consumer demands and the appliance industry is finding new and cheaper ways to manufacture appliances, many consumers do not wait until their appliances fail before replacing them. The marriage of information technology and appliance technology has great potential to entice consumers, but, as noted in the 
computer industry, the time to perceived obsolescence is often reduced. The result is an increase in production energy and disposal energy. In addition, maintenance issues may increase; therefore, appliances need to be designed for ease of maintenance, disassembly and recycling.

\section{References}

[1] Energy Information Administration, "2001 Residential Energy Consumption Survey: Household Energy Consumption and Expenditure Tables"

[2] Energy Policy and Conservation Act, Public Law 94-163, December 1975

[3] Energy Policy and Conservation Act, Public Law 95-619, November 1978

[4] Www.homeplug.org

[5] http://www.standards.ieee.org

[6] "Networking Consumer Devices," Hometoys Article, December 2001, Http://www.hometoys.com

[7] http://www.jeita.or.jp/english/public_standard/

[8] Echonet Specification, Version 2.1.1, Echonet Consortium, 2002, $\mathrm{http} / / / \mathrm{www}$. echonet.gr.jp/english/1_echo/index.htm

[9] http://www.ceced.org/sites/ceced.org/community/files/259/phpB0ifok/CHAINPR 1Eng.pdf

[10] AHAM CHA-1-2002, "Connected Home Appliances- Object Modeling," Association of Home Appliance Manufacturers

[11] http://www.epa.gov/fedrgstr/EPA-IMPACT/2003/March/Day-07/i5404.htm

[12] "Dishwashers, Power at a Price," Consumer Reports, March 2005 